\title{
Spatial and temporal pattern of expression of the cellular retinoic acid-binding protein and the cellular retinol-binding protein during mouse embryogenesis
}

(mammalian development/vitamin $\mathbf{A} /$ in situ hybridization/central nervous system)

\author{
Ana V. Perez-Castro*, Leslie E. Toth-Rogler ${ }^{\dagger}$, Li-na Wei ${ }^{\ddagger}$, and M. Chi Nguyen-Hud* \\ Departments of Microbiology and Urology, Columbia University College of Physicians and Surgeons, 701 West 168 th Street, New York, NY 10032
}

Communicated by Bruce M. Alberts, August 21, 1989

\begin{abstract}
Retinol (vitamin $A$ ) and retinoic acid are potent teratogens and also represent good candidates for normal morphogens during development. Their actions may be mediated by the cellular retinoic acid-binding protein (CRABP) and the cellular retinol-binding protein (CRBP). As a step towards understanding the possible function for CRABP and CRBP in morphogenesis, we have used in situ hybridization to analyze their expression during mouse development. Both CRABP and CRBP transcripts were detected at embryonic days 9.5-14.5. (i) In the nervous system, CRABP transcripts were found in the mantle layer of the dorsal spinal cord and hindbrain and in the marginal layer of the midbrain, whereas CRBP transcripts were found in the ependymal and mantle layer of the ventral spinal cord and of the forebrain as well as in the spinal nerves and the roof plate of the spinal cord. (ii) In the eye, CRABP is expressed in the retinal layer, and CRBP is expressed in both retinal and pigmented layers. (iii) In the craniofacial region, CRABP transcripts were found in the mesenchyme of the frontonasal mass and mandible, while CRBP transcripts were found in the mesenchyme of the nasolachrymal duct and surrounding the auditory vesicle. Two general conclusions can be made. First, all of the tissues that are known to be teratogenic targets of retinoic acid and retinol also express CRABP and CRBP transcripts. Second, the specific expression of CRABP and CRBP in numerous developing tissues indicates that these proteins may perform specific functions during morphogenesis of a broad variety of embryonic structures.
\end{abstract}

The retinoids exert profound effects on development and differentiation in a wide variety of systems (1-7). In the developing chick limb, retinoic acid has the ability to change the positional information of cells in a very precise manner: for example, application of retinoic acid to the side opposite the zone of polarizing activity causes digit pattern duplication $(8,9)$. Retinoid treatment of chicken embryos affects the development of the upper beak by inhibiting the outgrowth and differentiation of the frontonasal mass (10).

In mammals, it is known that maternal hypervitaminosis $\mathrm{A}$ is responsible for a variety of congenital malformations (11). In mouse embryos, it has been reported that hypervitaminosis $\mathrm{A}$ causes alterations in the cell cycle of neuroepithelial cells and prevents the closure of the neural tube, producing brain deformities $(12,13)$. Excess vitamin A also alters the pattern of migration of cranial neural crest cells, producing facial skeleton deformities (cleft palate) $(14,15)$. Alterations in the cephalic mesoderm, the axial nonsegmented mesoderm, and finally the segmented mesoderm, especially the somites (rib defects and vertebral malformations), are also caused by administration of excess vitamin $A(16,17)$. In humans,

The publication costs of this article were defrayed in part by page charge payment. This article must therefore be hereby marked "advertisement" in accordance with 18 U.S.C. $\$ 1734$ solely to indicate this fact. maternal exposure to isotretinoin (Accutane), a retinoid derivative, has been reported to produce several malformations including craniofacial deformities (18). The teratogenic effects of exogenous retinoic acid may reflect a possible role for this compound as an endogenous morphogen in development. Indeed, retinoic acid has been detected in the developing chick limb forming a gradient across the bud (19).

The molecular mechanisms by which retinoids affect morphogenesis are not understood. It is possible that their action is mediated by some of the known intracellular proteins that specifically bind retinoic acid or retinol. There exist an intracellular protein that binds retinoic acid, the cellular retinoic acid-binding protein (CRABP), and an intracellular protein that binds retinol, the cellular retinol-binding protein (CRBP) $(20,21)$. Nuclear retinoic acid receptors have also been described: the nuclear retinoic acid receptors $\alpha$ and $\beta$ $(22,23)$. These nuclear receptors act as transcriptional activators in a retinoic acid-dependent manner. It has been proposed that CRABP and the nuclear retinoic acid receptors may cooperate in the mechanism of action of retinoic acid (24). Interestingly, CRABP has been detected in the developing chicken limb and forms a gradient that is opposite in direction to the gradient formed by retinoic acid (25).

As a step towards understanding the function of CRABP and CRBP in embryogenesis, we have used in situ hybridization to define the pattern of expression of these two genes. Our data show that CRABP and CRBP transcripts accumulate in specific temporal and spatial patterns, indicating a specific function for these genes during morphogenesis.

\section{MATERIALS AND METHODS}

Plasmids. The plasmids pLNWX and pLNWY were constructed by subcloning in both orientations (sense and antisense) a 400-base-pair (bp) Alu I-Alu I fragment containing exon 1 of the mouse CRABP gene into the EcoRI site of pGem-2. The complete mouse CRABP gene has been isolated in our laboratory as a cosmid and contains four exons (unpublished data; refs. 26 and 27 ). The plasmid pG2AVP was constructed by subcloning a 500-bp EcoRI-Pst I fragment containing exon 4 of the mouse CRBP gene into the EcoRI/Pst I sites of pGem-2. The mouse CRBP gene has been isolated in our laboratory as a $\lambda$ recombinant phage and contains four exons (unpublished data; refs. 26 and 28 ).

Preparation of Mouse Embryo Sections. C57BL/6J inbred mice were mated and examined the next morning for the presence of a vaginal plug. This was considered to be 0.5 day

Abbreviations: CRABP, cellular retinoic acid-binding protein; CRBP, cellular retinol-binding protein; CNS, central nervous system.

*Present address: Department of Microbiology, University of Southern California, 1441 Eastlake Avenue, Los Angeles, CA 90033.

†Present address: Department of Anatomy and Cell Biology, Columbia University, New York, NY 10027.

$\ddagger$ Present address: Department of Microbiology, Chang Gung Medical College, Kwei-San, Tao-Yuan, Taiwan. 
postcoitum (p.c.). Embryos were isolated at different days of gestation: $3.5,7.5,8.5,9.5,10.5,11.5,12.5,14.5$ days p.c. and fixed for $24 \mathrm{hr}$ in $4 \%$ paraformaldehyde in phosphatebuffered saline (PBS) at $4^{\circ} \mathrm{C}$. After fixation the embryos were placed in $0.5 \mathrm{M}$ sucrose in PBS at $4^{\circ} \mathrm{C}$ for $24 \mathrm{hr}$ and embedded in OTC compound (Miles). Sections were cut $(8-10 \mu \mathrm{m})$ and placed on poly(L-lysine)-coated slides and stored at $-70^{\circ} \mathrm{C}$.

Preparation of Single-Stranded RNA Probes. pLNWX and pLNWY were linearized with HindIII, whereas pG2AVP was linearized in two different ways: for the sense probe (same sequence as transcript) with $P$ st I and for the antisense probe (sequence complementary to transcript) with EcoRI.

${ }^{35} \mathrm{~S}$-labeled probes with a specific activity of $1-2 \times 10^{9}$ $\mathrm{cpm} / \mu \mathrm{g}$ were prepared for plasmids $\mathrm{pLNWX}$ (sense CRABP probe), pLNWY (antisense CRABP probe), and pG2AVP linearized with Pst I by using Sp6 RNA polymerase (sense CRBP probe) and for pG2AVP linearized with EcoRI by using T7 RNA polymerase (antisense CRBP probe).

In Situ Hybridization. In situ hybridization was performed as described (29). After dipping the slides in photographic emulsion NTB-2 (Kodak), they were exposed for 1-2 weeks, developed, and stained with Harris's hematoxylin/eosin.

\section{RESULTS}

Spatial Expression of CRABP and CRBP Transcripts. To determine if CRABP is expressed during mouse embryogenesis, we performed in situ hybridization initially with frontal sections of a 10.5-day embryo. In the representative section shown in Fig. 1, CRABP transcripts are found in the limb bud (lb), the frontonasal mass (fnm), and the spinal cord (sc) and myelencephalon (mye). In the limb bud, a gradient pattern was observed along the anteroposterior axis, with the highest concentration at the anterior side. Thus, the CRABP transcript distribution in the limb is similar to the CRABP protein distribution reported by Maden et al. (25). In the frontonasal mass, expression was seen in the mesenchymal cells but not in the epithelial cells. In the spinal cord and myelencephalon, expression was seen in the mantle layer but not in the ependymal layer. Furthermore, expression in the spinal cord was restricted mainly to the dorsal part.

To extend the CRABP data obtained with 10.5-day embryos and to determine whether CRBP is also expressed, we hybridized a series of adjacent sagittal sections of an 11.5-day mouse embryo and with either the CRABP or the CRBP probe (Fig. 2 Upper). The CRABP pattern observed was entirely consistent with the results presented in Fig. 1. Moreover, we detected CRABP transcripts in additional places such as the mandible, the mesencephalon, the liver, the heart ventricle, the tongue, and the cells surrounding the dorsal aorta. Expression around the dorsal aorta seemed to be confined to the surrounding mesoderm and not to endothelial cells (see Fig. $4 c$ and $c^{\prime}$ ).

Fig. 2 shows that CRBP transcripts were also present in a defined pattern. Intense hybridization was seen in the ventral part of the spinal cord, the tongue, the liver, and around the stomach (Fig. 2 Upper). Hybridization grains were also seen in the lung, around the large intestine, and the Wolffian and Mullerian ducts (data not shown). Hybridization was further detected in the dorsal root ganglia of the cervical region, in some cranial ganglia, and in the spinal nerves (see Fig. $4 f$ and $\left.f^{\prime}\right)$. However, the intensity of hybridization in the ganglia was weaker than in the spinal cord. Hybridization grains were also abundant in the telencephalon, the myelencephalon, and the mesenchyme surrounding the auditory and optic vesicles.

To extend these observations, we further analyzed transverse sections of a 12.5-day mouse embryo (Fig. 2 Lower). The patterns of both CRABP and CRBP transcripts showed good correlation with the patterns already described for the 10.5- and 11.5-day embryos. In the limb bud, CRABP transcripts were found in the lateral mesenchyme but not in the medial mesenchyme. In addition, a proximodistal gradient can be observed, with the highest concentration at the proximal side.

Expression in the Central Nervous System (CNS) Is Cell Specific. The data in Figs. 1 and 2 show that expression of CRABP and CRBP in the CNS is specific to certain cell types. Therefore, we examined our sections under a high-power microscope (Fig. 3).

In the spinal cord, CRABP was expressed in the dorsally located alar plates and in the intermediate horn, specifically in the mantle layer (Fig. $3 a$ and $a^{\prime}$ ). On the other hand, CRBP was expressed in the ependymal and mantle layers of the ventrally located basal plates and in the roof plate (Fig. $3 e$ and $\left.e^{\prime}\right)$. The basal and alar plates will give rise to motor and sensory neurons respectively, whereas the intermediate horn will give rise to mainly neurons of the autonomic nervous
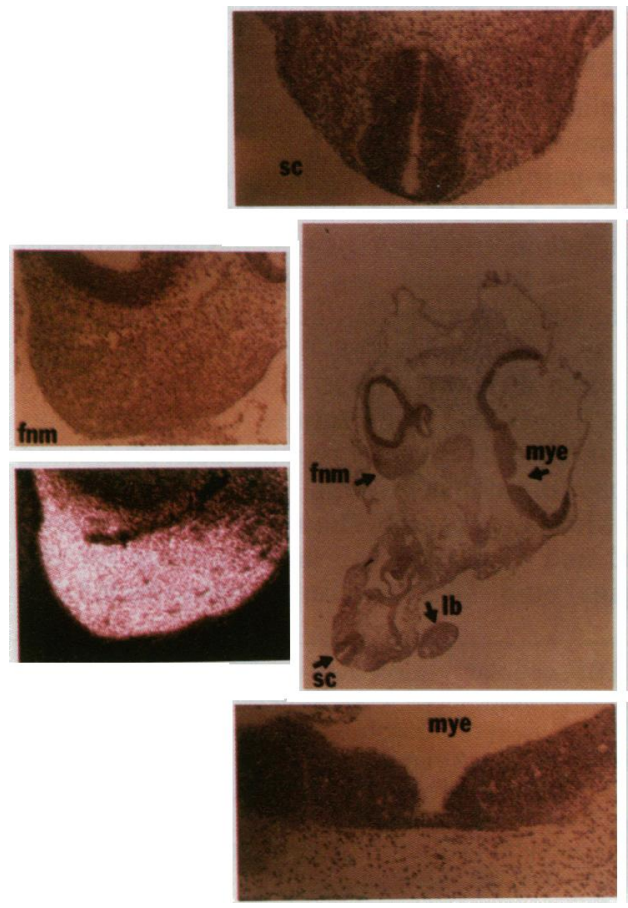
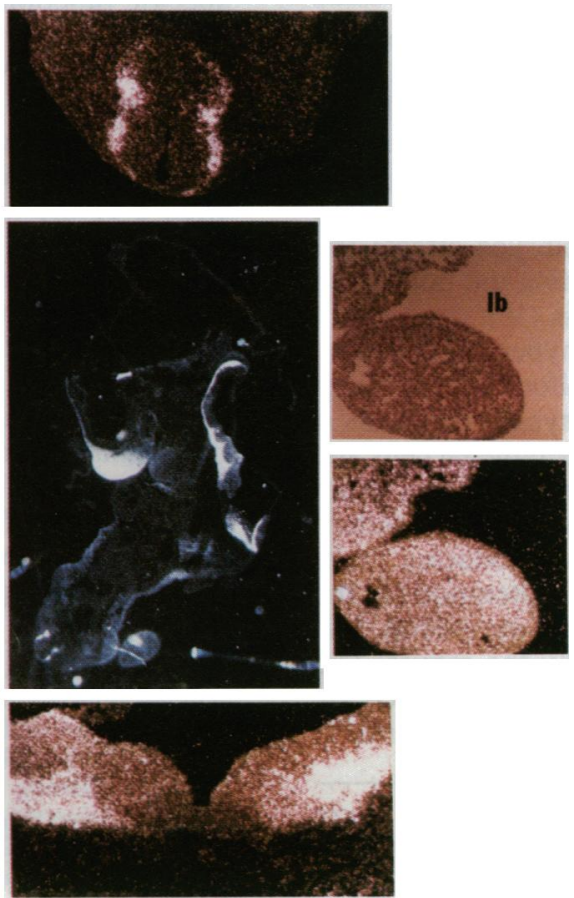

Fig. 1. Localization of CRABP transcripts in a 10.5-day mouse embryo. Brightfield (Left Center) and dark-field (Right Center) low-power photomicrographs of a frontal section of a 10.5-day mouse embryo hybridized with an antisense CRABP probe and stained with hematoxylin/eosin are shown as the two larger centrally located photomicrographs. Arrows in the brightfield photomicrograph indicate areas of hybridization, which are also shown at a higher magnification. Bright-field (Left) and darkfield (Right) high-power photomicrographs (smaller photomicrographs surrounding the larger ones) of these areas are shown for the spinal cord (sc) (Top), the myelencephalon (mye) (Bottom), the frontonasal mass (fnm) (Left), and the limb bud (lb) (Right). The plane of sectioning through the limb is perpendicular to the proximodistal axis and contains the anteroposterior and dorsoventral axes: anterior at the top, posterior at the bottom, dorsal at the left, and ventral at the right. 

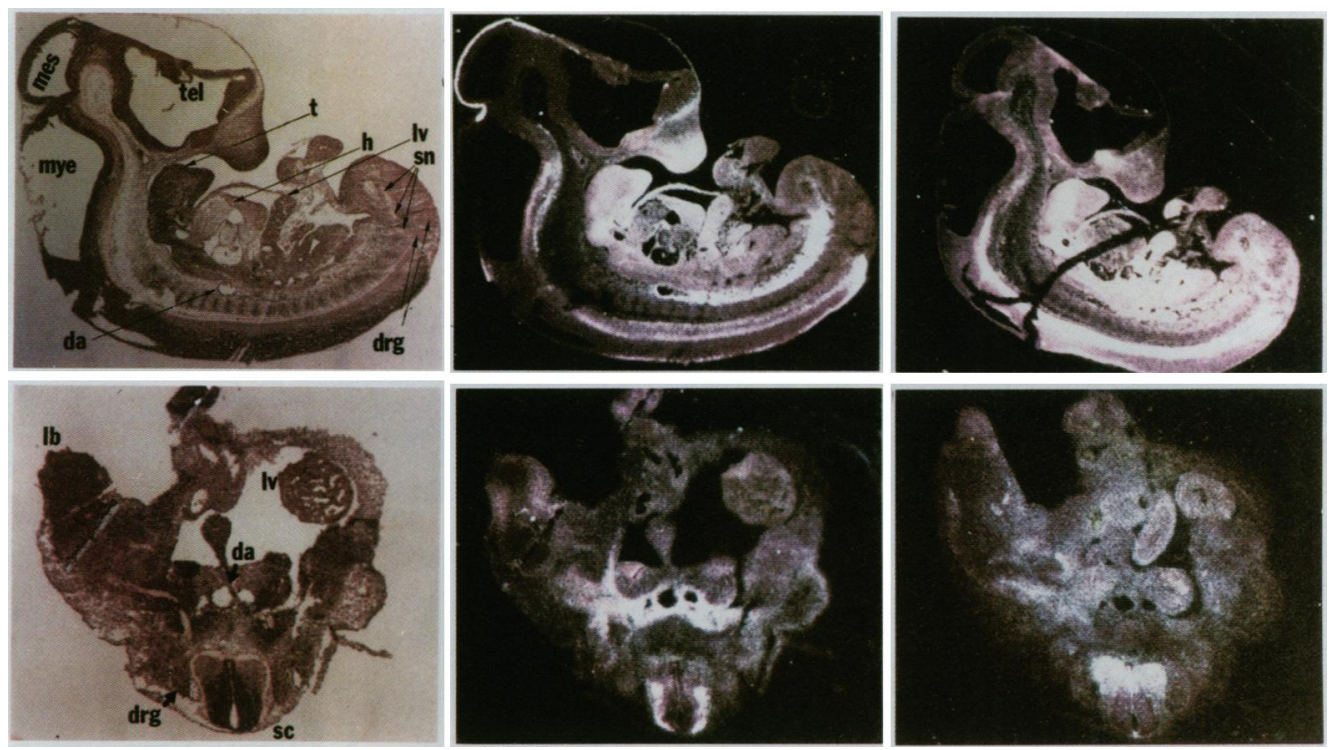

Fig. 2. Localization of CRABP and CRBP transcripts in 11.5- and 12.5-day mouse embryos. (Upper) Sagittal sections of an 11.5-day embryo. (Upper Left) Bright-field photomicrograph on which arrows specify certain areas: tel, telecephalon; mes, mesencephalon; mye, myelencephalon; sc, spinal cord; drg, dorsal root ganglia; sn, spinal nerves; h, heart; t, tongue; lv, liver; da, dorsal aorta. (Upper Center) Dark-field photomicrograph of a section hybridized with the CRABP probe. (Upper Right) Dark-field photomicrograph of an adjacent section hybridized with the CRBP antisense probe. (Lower) Transverse sections of a 12.5-day embryo. (Lower Left) Bright-field photomicrograph on which arrows specify certain areas designated as above plus lb for limb bud. (Lower Center) Dark-field photomicrograph of a section hybridized with the CRABP antisense probe. (Lower Right) Dark-field photomicrograph of an adjacent section hybridized with the CRBP antisense probe.

system. On the other hand, the dorsal and ventral midline portions of the spinal cord, known as the roof and floor plates, do no: contain neuroblasts and serve primarily as pathways for nerve fibers.

In the brain, CRABP transcripts present in the mesencephalon were located at the marginal layer (Fig. $3 c$ and $c^{\prime}$ and $d$ and $\left.d^{\prime}\right)$, but transcripts in the myelencephalon and metencephalon were found in the mantle layer (Fig. $3 b$ and $b^{\prime}$ ). CRBP expression in the telencephalon on the other hand, seemed to be confined to its anterior basal portion and to the ependymal and mantle layers (Figs. $3 e$ and $e^{\prime}$ and $f$ and $f^{\prime}$ ), whereas in the myelencephalon, transcripts were detected in the mantle layer (data not shown). Expression of CRABP and CRBP in the CNS is summarized in Table 1.

CRABP and CRBP Transcripts Are Present in the Sensory Organs. We have already seen that CRABP was expressed in the frontonasal mass at day 10.5. Later on, at day 12.5, CRABP was still expressed in this area, but by then the nasal chambers had formed and CRABP transcripts were found in the mesenchyme but not in the olfactory epithelium (Fig. $4 b$ and $\left.b^{\prime}\right)$. Similarly, at 12.5 days, CRBP transcripts were present around the nasolachrymal duct, specifically in the mesenchyme and not in the epithelial cells (data not shown). In the auditory vesicle, CRBP transcripts were found specifically in the mesodermal cells (Fig. $4 e$ and $e^{\prime}$ ), whereas CRABP transcripts were not detected. In the eye, CRABP and CRBP transcripts were found in the differentiating retinal layer (Fig. $4 a, a^{\prime}$, and $a^{\prime \prime}$ ). Furthermore, CRBP was also expressed in the pigmented layer (Fig. $4 d$ and $d^{\prime}$ ), where vitamin A uptake occurs.

Temporal Expression of CRABP and CRBP Transcripts. To examine any temporal changes of the spatial pattern, we hybridized a series of sections from embryos of different ages: $3.5,7.5,8.5,9.5,10.5,11.5,12.5$, and 14.5 days. No hybridization was observed with 3.5-day blastocysts and 7.5- and 8.5-day embryos. In contrast, strong hybridization was observed in 9.5-day embryos with both probes (data not shown).

Thus, at 9.5 days, CRABP is expressed in the telencephalon, mesencephalon, metencephalon, myelencephalon, the spinal cord, and also in the heart bulge. The pattern observed in the spinal cord is similar to the one observed in older embryos (10.5-12.5 days). On the other hand, CRBP seemed to be expressed in many other places than the CNS, such as the gut, the heart, and the liver. In the CNS, labeling with CRBP was seen in the very front end of the telencephalon, in the boundary between the diencephalon and mesencephalon, in the cells surrounding the fourth ventricle, and in the spinal cord. The pattern of expression in the spinal cord at day 9.5 was similar to that observed for older embryos (10.5-12.5 days). It is interesting that both transcripts first appeared at day 9.5 , at the time when organogenesis is greatly accelerated and the contour of the embryo is being altered significantly.

The pattern of expression of CRABP and CRBP between 10.5 and 12.5 days has already been described (Figs. 1-4). At day 14.5, there was still tissue-specific expression of CRABP and CRBP transcripts (data not shown). For CRBP, transcripts are present in the CNS (brain), eyes, liver, heart, lungs, and limbs. For CRABP, there is expression of transcripts in the kidney, liver, lung, CNS, heart, limb, and epidermis.

\section{DISCUSSION}

We have defined the spatial and temporal pattern of expression of CRABP and CRBP during mouse embryogenesis.

The tissue distribution of CRABP and CRBP transcripts that we have found are in good correlation with the spectrum of tissues affected teratogenically by retinoic acid and retinol in embryos of different species $(1,12-15,30-34)$. CRABP and CRBP transcripts were found in the limb, spinal cord, brain, and facial structures, which all are well-known teratogenic targets of retinoic acid and retinol. Moreover, this correlation seems to be extended to the level of cell specificity. For example, the mesenchyme of the frontonasal mass rather than the ectoderm is specifically the target of retinoid action in chicken embryos (10). Again, we found that expression of CRABP transcripts are present in the mesoderm and not in the epithelial cells of the frontonasal mass. Teratogenicity caused by retinoic acid and retinol is strongly dependent on the development stage of the exposed embryos. In the developing limb, Kochhar (31) reported that at earlier stages retinoic acid disrupts cellular activity of the mesenchymal 

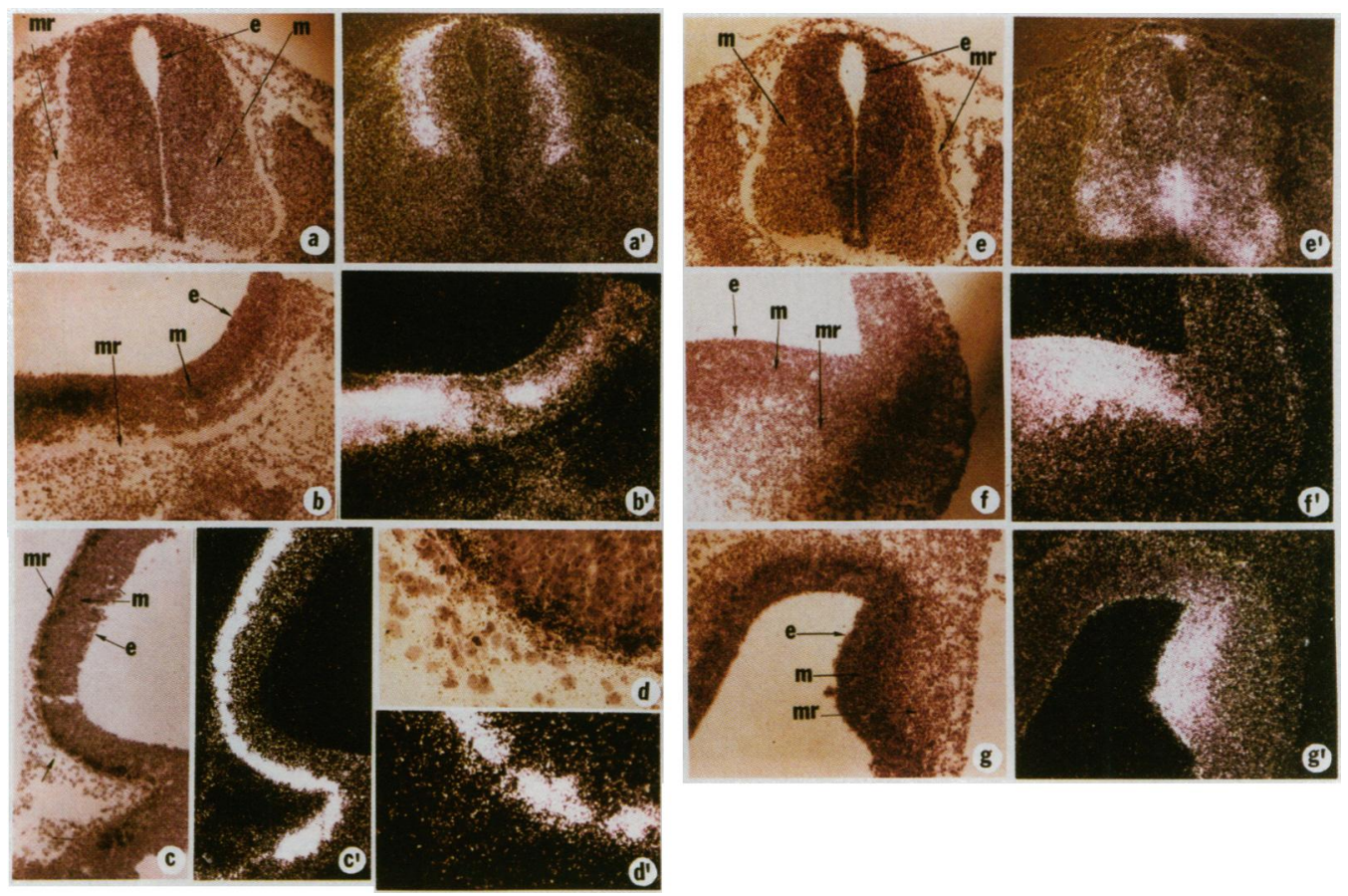

FIG. 3. Distribution of CRABP and CRBP transcripts in the CNS. (a) Transverse section of a 12.5-day embryo, showing the spinal cord and its different layers: e, ependymal; $\mathrm{m}$, mantle; $\mathrm{mr}$, marginal layers. $\left(a^{\prime}\right)$ Dark-field photomicrograph of the same section hybridized to the CRABP probe. Hybridization is detected in the dorsolateral mantle layer. (b) Sagittal section of an 11.5-day embryo, showing the myelencephalon and its different layers. $\left(b^{\prime}\right)$ Dark-field photomicrograph of the same section hybridized to the CRABP probe. Hybridization grains are in the mantle layer. (c) Sagittal section of an 11.5-day embryo, showing the mesencephalon with its different layers. $\left(c^{\prime}\right)$ Dark-field photomicrograph of the same section probed with CRABP. $\left(d\right.$ and $\left.d^{\prime}\right)$ Higher magnification of $c$ and $c^{\prime}$, showing that high expression is localized in the marginal layer. The portion magnified is shown by an arrow in $c$. (e) Transverse section of a 12.5-day embryo, showing the spinal cord and its different layers. $\left(e^{\prime}\right)$ Dark-field photomicrograph of the same section hybridized to the CRBP probe. Labeling is found in the ependymal and mantle layers of the ventral spinal cord. $(f)$ Sagittal section of an 11.5-day embryo, showing the telencephalon and its different layers. $\left(f^{\prime}\right)$ Dark-field photomicrograph of the same section hybridized with a CRBP probe. Hybridization is confined only to the basal anterior portion of the telencephalon and to its ependymal and mantle layers. $(g)$ Transverse section of a 12.5-day embryo, also showing the telencephalon and its different layers. $\left(g^{\prime}\right)$ Dark-field photomicrograph of the same section hybridized to a CRBP probe.

condensations, and at later stages it prevents the enlargement of the condensations that have already appeared. In cleft palate incidence caused by retinoids, Shenefelt $(35)$ reported a correlation between the type of malformation caused by retinoic acid in hamster embryos and the time of treatment. Again, the stages at which we observed CRABP and CRBP expression corresponded well with the stages at which the embryos are susceptible to the teratogenicity of retinoids. Thus, both the spatial and temporal pattern of CRABP and CRBP expression are entirely consistent with the possibility that the teratogenic effects of retinoic acid and retinol are being mediated by CRABP and CRBP.
What are the normal roles of CRABP and CRBP in embryogenesis? We considered it likely that CRABP and CRBP would be involved in the possible morphogenic action of endogenous retinoids. For example in the developing limb, a gradient of retinoic acid has been found along the anteroposterior axis, the highest concentration being on the posterior side, which is the site of the zone of polarizing activity (19). A reciprocal gradient has been found for CRABP (25). Maden et al. have proposed that these two gradients would result in a flatter distribution of the retinoic acid-CRABP complex across the limb bud, but in a steeper concentration gradient of free retinoic acid (25). Thus, free retinoic acid would then act

Table 1. Temporal and spatial expression of CRABP and CRBP transcripts in the CNS

\begin{tabular}{|c|c|c|c|c|}
\hline & \multicolumn{4}{|c|}{ Transcripts in embryo CNS } \\
\hline & \multicolumn{2}{|c|}{9.5 days } & \multicolumn{2}{|c|}{$10.5-12.5$ days } \\
\hline & CRABP & CRBP & CRABP & CRBP \\
\hline Telencephalon & + & + & - & + (epend., mantle) \\
\hline Diencephalon & - & $+^{*}$ & - & - \\
\hline Mesencephalon & + & $+^{*}$ & $+($ marginal $)$ & - \\
\hline Metencephalon & + & - & $+($ mantle $)$ & - \\
\hline Myelencephalon & + & + & $+(\operatorname{mantle})$ & $+(\operatorname{mantle})$ \\
\hline Spinal cord & + & + & $\begin{array}{l}+ \text { (mantle } \\
\quad \text { alar plate })\end{array}$ & $\begin{array}{l}+ \text { (epend., mantle, } \\
\text { basal and roof plates) }\end{array}$ \\
\hline
\end{tabular}

,+ CNS structures in which CRABP or CRBP transcripts are found. In parentheses are specified the layers of neuroepithelial cells where expression occurs. The inner layer [ependymal (epend.)] is constituted by mitotically dividing neuroepithelial cells, the middle layer (mantle) by nondividing neuroblasts that later will form the gray matter, and the outermost layer (marginal) by nerve fibers emerging from the neuroblasts, which will form the white matter. For the spinal cord, we have additionally indicated the plates that express the transcripts.

*Transcripts are expressed at the boundary of these two structures. 

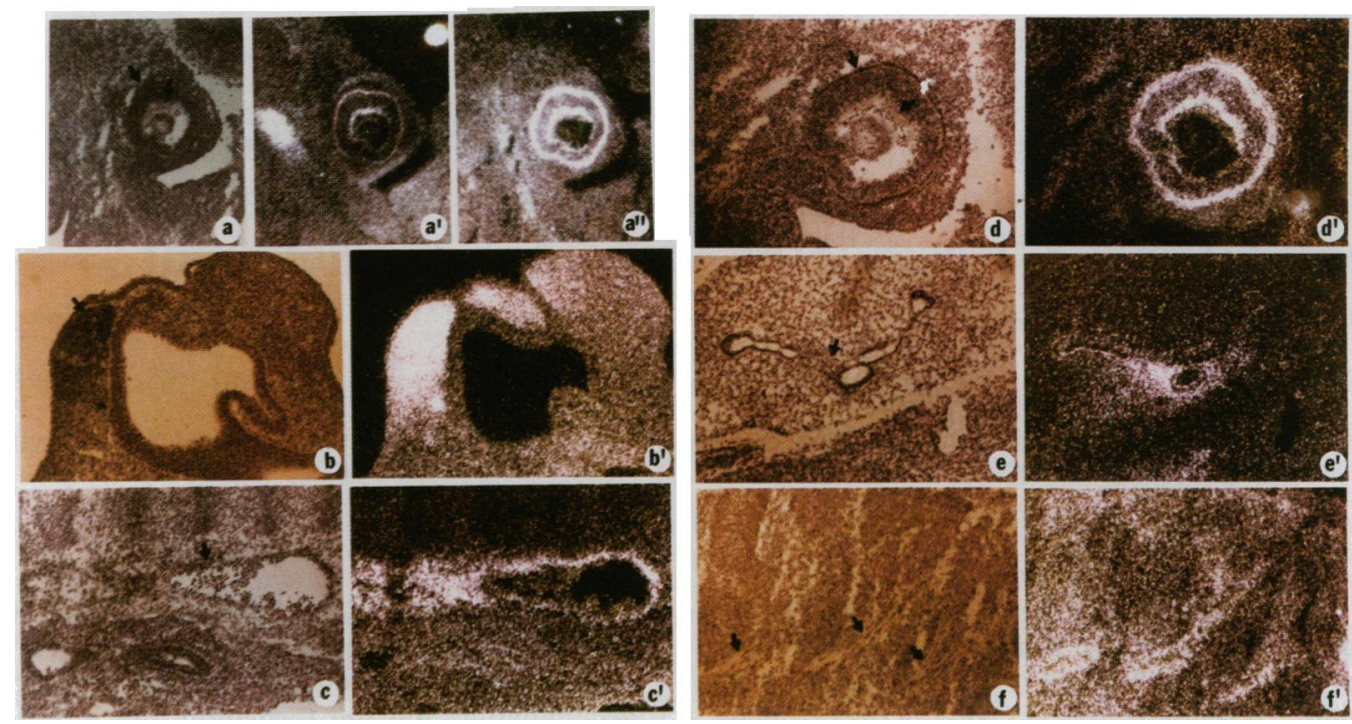

FIG. 4. Distribution of CRABP and CRBP transcripts in specific tissues. (a) Sagittal section showing the eye of an 11.5-day embryo. ( $a^{\prime}$ ) Dark-field photomicrograph of the same section hybridized with the CRABP probe. Strong hybridization is detected in the retinal layer. On the other hand, the weak hybridization seen in the pigmented layer with the CRABP probe is an artifact, since this was also found with a negative control probe. $\left(a^{\prime \prime}\right)$ Dark-field photomicrograph of an adjacent section hybridized with the CRBP probe. Grains are found in the pigmented and retinal layers. (b) Sagittal section of an 11.5-day embryo, showing tissues around the nasal chamber. ( $\left.b^{\prime}\right)$ Dark-field photomicrograph of the same section hybridized with the CRABP probe. Hybridization is specifically found in cells from the mesenchyme. (c) Sagittal section of an 11.5-day embryo, showing the dorsal aorta. $\left(c^{\prime}\right)$ Dark-field photomicrograph of the same section hybridized to the CRABP probe. Grains are detected only in cells derived from the mesoderm. $\left(d\right.$ and $\left.d^{\prime}\right)$ Higher magnification of the eye section in $a^{\prime \prime}:$ Bright-field photomicrograph $(d)$ and dark-field photomicrograph $\left(d^{\prime}\right)$. (e) Transverse section of a 12.5-day embryo, showing the developing otic vesicle. $\left(e^{\prime}\right)$ Dark-field photomicrograph of the same section hybridized with the CRBP probe. Hybridization was confined to the surrounding mesoderm. $(f)$ Sagittal section of an 11.5-day embryo, showing three spinal nerves. $\left(f^{\prime}\right)$ Dark-field photomicrograph of the same section probed with CRBP. Hybridization was found in the spinal nerves. Arrows in bright-field panels denote the area of hybridization.

as a morphogen to alter patterns of gene transcription, by means of the nuclear retinoic acid receptor that is likely to be present at equal concentration throughout the limb (24). Our findings of CRABP and CRBP expression in the dorsal and ventral areas of the spinal cord, respectively, may point to a similar model for the developing CNS. It is known that the dorsal and ventral areas will acquire sensory and motor characteristics, respectively. The high expression of CRBP in the ventral area may serve to concentrate retinol from the blood stream to this area of the CNS, where retinol will be converted into retinoic acid. Retinoic acid then may form a concentration gradient along the dorsoventral axis, being highest on the ventral side. The gradient of free retinoic acid may be further steepened by the presence of CRABP in the dorsal area, and this gradient may be crucial for the differentiation of sensory and motor neurons. This model can be tested for example by altering the pattern of expression of CRABP and CRBP in the developing CNS of transgenic mice. Regardless of the mechanism of action of CRABP and CRBP, the normal patterns of expression defined here will be useful in future experiments directed at understanding the function of these highly conserved proteins.

We thank Drs. Jane Dodd, John Pintar, and Charles Rogler for kindly sharing with us their equipment. We thank Dr. John Pintar for critically commenting on the manuscript. This work was supported by a Grant from the National Institutes of Health (ROIHD 19821).

1. Kochhar, D. M., Penner, J. D. \& Tellone, C. I. (1984) Teratogen. Carcinogen. Mutagen. 4, 377-387.

2. Bolmer, S. D. \& Wolf, G. (1982) Proc. Natl. Acad. Sci. USA 79, 6541-6545.

3. Fuchs, E. \& Green, H. (1981) Cell 25, 617-625.

4. Pennypacker, J. P., Lewis, C. A. \& Hassell, J. R. (1978) Arch. Biochem. Biophys. 186, 351-358.

5. Jetten, A. M., Jetten, M. E. R. \& Sherman, M. I. (1979) Exp. Cell. Res. 124, 381-391.

6. Wei, L., Blaner, W. S., Goodman, D. S. \& Nguyen-Huu, M. C. (1989) Mol. Endocrinol. 3, 454-463.
7. Abbott, B. D., Adamson, E. D. \& Pratt, R. M. (1988) Development 102, 853-867.

8. Maden, M. \& Summerbell, D. (1986) J. Embryol. Exp. Morphol. 97 , 239-250.

9. Tickle, C., Alberts, B., Wolpert, L. \& Lee, J. (1982) Nature (London) 296, 564-566.

10. - Wedden, S. E. (1987) Development 99, 341-351.

11. Kwarta, R. F., Kimmel, C. A., Jr., Kimmel, G. L. \& Slikker, W., Jr. (1985) Teratology 32, 103-111.

12. Langman, J. \& Welch, G. W. (1966) J. Comp. Neurol. 128, 1-16.

13. Langman, J. \& Welch, G. W. (1967) J. Comp. Neurol. 131, 15-26.

14. Wiley, M. J., Cauwenbergs, P. \& Taylor, I. M. (1983) Acta Anat. 116, 180-192.

15. Yasuda, Y., Okamoto, M., Konishi, H., Matsuo, T., Kihara, T. \& Tanimura, T. (1986) Teratology 34, 37-49.

16. Marin-Padilla, M. \& Ferm, V. H. (1965) J. Embryol. Exp. Morphol. 13, 1-8.

17. Marin-Padilla, M. (1966) J. Embryol. Exp. Morphol. 15, 261-269.

18. Rosa, F. W. (1984) Lancet ii, 513.

19. Thaller, C. \& Eichele, G. (1987) Nature (London) 327, 625-628.

20. Ong, D. E. \& Chytil, F. (1978) J. Biol. Chem. 253, 828-832.

21. Ong, D. E. \& Chytil, F. (1978) J. Biol. Chem. 253, 4551-4554.

22. Petkovich, M., Brand, N. J., Krust, A. \& Chambon, P. (1987) Nature (London) 330, 444-450.

23. Giguere, V., Ong, E. S., Segui, P. \& Evans, R. M. (1987) Nature (London) 330, 624-629.

24. Giguere, V., Ong, E. S., Evans, R. M. \& Tabin, C. J. (1989) Nature (London) 337, 566-569.

25. Maden, M., Ong, D. E., Summerbell, D. \& Chytil, F. (1988) Nature (London) 335, 733-735.

26. Wei, L., Mertz, J. R., Goodman, D. S. \& Nguyen-Huu, M. C. (1987) Mol. Endocrinol. 1, 526-534.

27. Shubeita, H. E., Sambrook, J. F. \& McCormick, A. M. (1987) Proc. Natl. Acad. Sci. USA 84, 5645-5649.

28. Nilsson, M. H. L., Spurr, N. K., Lundvall, J., Rask, L. \& Peterson. P. A. (1988) Eur. J. Biochem. 173, 35-44.

29. Toth, L. E., Slawin, K. L., Pintar, J. E. \& Nguyen-Huu, M. C. (1987) Proc. Natl. Acad. Sci. USA 84, 6790-6794.

30. Wiley, M. J. (1983) Teratology 28, 341-353.

31. Kochhar, D. M. (1973) Teratology 7, 289-298.

32. Taylor, I. M., Wiley, M. J. \& Agur, A. (1980) Teratology 21, 193-197.

33. Morriss, G. M. \& Steele, C. E. (1976) Teratology 15, 109-120.

34. Newall, D. R. \& Edwards, J. R. G. (1981) Teratology 23, 125-130.

35. Shenefelt, R. E. (1972) Teratology 5, 103-118. 